\title{
DIGITALCOMMONS
}

@WAYNESTATE —

Wayne State University

9-1-2002

\section{A Posteriori Error Estimates Based on Polynomial Preserving Recovery}

Zhimin Zhang

Wayne State University, zhimin.zhang@wayne.edu

Ahmed Naga

Wayne State University

\section{Recommended Citation}

Zhang, Zhimin and Naga, Ahmed, "A Posteriori Error Estimates Based on Polynomial Preserving Recovery" (2002). Mathematics Research Reports. Paper 6.

http://digitalcommons.wayne.edu/math_reports/6

This Technical Report is brought to you for free and open access by the Mathematics at DigitalCommons@WayneState. It has been accepted for inclusion in Mathematics Research Reports by an authorized administrator of DigitalCommons@WayneState. 


\title{
A POSTERIORI ERROR ESTIMATES BASED ON POLYNOMIAL PRESERVING RECOVERY
}

\author{
ZHIMIN ZHANG and AHMED NAGA
}

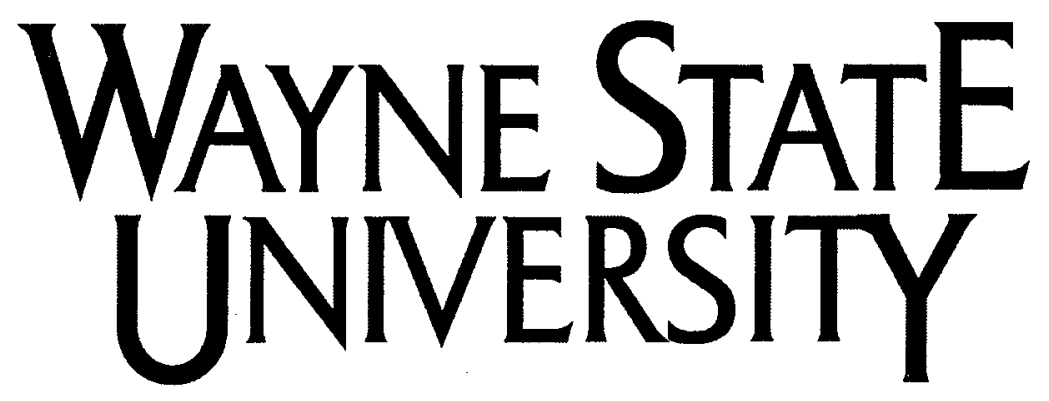

Detroit, MI 48202

Department of Mathematics

Research Report

\section{Series}

\#9

This research was partly supported by the National Science Foundation. 


\title{
A Posteriori Error Estimates Based on Polynomial Preserving Recovery
}

\author{
Zhimin Zhang*and Ahmed Naga \\ Department of Mathematics, Wayne State University
}

\begin{abstract}
Superconvergence of order $O\left(h^{1+\rho}\right)$, for some $\rho>0$, is established for gradients recovered using Polynomial Preserving Recovery technique when the mesh is mildly structured. Consequently this technique can be used in building a posteriori error estimator that is asymptotically exact.
\end{abstract}

Key Words. finite element method, least-squares fitting, SPR, PPR, superconvergence

AMS Subject Classification. 65N30, 65N15, 65N12, 65D10, 74S05, 41A10, 41A25

\section{Introduction}

Adaptive control based on a posteriori error estimates have become standard in finite element methods. Generally speaking, error estimators can be classified under two categories. The first one is the residual type estimators, as in [4], and the second one is the recovery type estimators, as in [12]. In recovery type estimators, a recovery operation uses the finite element solution or its gradient to build another solution, as in [7] and [9], or another gradient, as in [13]. The recovered quantities are then used in building a posteriori error estimators (see [1] and [3] for some general discussion and literature).

As it is known, if the recovered gradient is superconvergent to the exact gradient, then the a posteriori error estimator based on this recovered gradient is exact in asymptotic sense. A good example of such estimators is Zienkiewicz-Zhu error estimator based on Superconvergence Patch Recovery (SPR), as in [14]. The Polynomial Preserving Recovery (PPR) is a new recovery technique, introduced in [11], which has good properties that enable it to be used in constructing a posteriori error estimator.

To fix the ideas, let $\Omega \subset \mathbb{R}^{2}$ be a bounded domain with Lipschitz boundary $\partial \Omega$. Consider

*This research was partially supported by the National Science Foundation grants DMS-0074301, DMS0079743 , and INT-0196139. 
the model elliptic boundary value problem of finding the solution $u$ of

$$
-\nabla(\mathcal{D} \nabla u+b u)+c u=f \text { in } \Omega
$$

subject to the boundary conditions

$$
n \cdot(\mathcal{D} \nabla u+b u)=g \text { on } \Gamma_{N}
$$

and

$$
u=0 \text { on } \Gamma_{D}
$$

Here $\mathcal{D}$ is a $2 \times 2$ symmetric positive definite matrix with smooth entries. The rest of the data are assumed to be smooth, $c$ is a non-negative function, $n$ is the unit outward normal vector to $\partial \Omega$, and the boundary segments are assumed to be disjoint with $\bar{\Gamma}_{D} \cup \bar{\Gamma}_{D}=\partial \Omega$. As usual, $W_{p}^{m}(\Omega)$ and $H^{m}(\Omega)$ are the classical Sobolev spaces equipped with the norms \|\|$_{m, p, \Omega}$, and \|\|$_{m, \Omega}$, respectively.

The variational form of this problem is to find $u \in V$ such that

$$
B(u, v)=L(v) \text { for all } v \in V
$$

where

$$
\begin{gathered}
V=\left\{v \in H^{1}(\Omega): v=0 \text { on } \Gamma_{D}\right\}, \\
B(u, v)=\int_{\Omega}[(\mathcal{D} \nabla u+\boldsymbol{b u}) \nabla v+c u v] d x d y,
\end{gathered}
$$

and

$$
L(v)=\int_{\Omega} f v d x+\int_{\Gamma_{N}} g v d s .
$$

Let $\mathcal{T}_{h}$ be a triangular partition of $\Omega$, and let $\Omega_{h}=\bigcup_{T \in \mathcal{T}_{h}} T$. Consider the $C^{0}$ linear finite element space $S_{h} \subset V$ associated with $\mathcal{T}_{h}$ and defined by

$$
S_{h}=\left\{v \in V: v \in P_{1}(T) \text { for every triangle } T \in \mathcal{T}_{h}\right\}
$$

where $P_{r}(\mathcal{A})$ denotes the set of all polynomials defined on $\mathcal{A} \subseteq \mathbb{R}^{2}$ of total degree $\leq r$. The finite element solution of this problem is to find $u_{h}$ such that

$$
B\left(u_{h}, v\right)=L(v) \text { for all } v \in S_{h}^{0}
$$

where

$$
S_{h}^{0}=\left\{v \in S_{h}:\left.v\right|_{\Gamma_{D}}=0\right\} .
$$


Unfortunately, $\nabla u_{h}$ is piecewise continuous, and so a smoothing operation is needed to get a continuous gradient of the solution. Recovery techniques like SPR and PPR can be used for this purpose. Both of them recover the gradient at mesh nodes. This is enough to uniquely define $R_{h} u_{h} \in S_{h} \times S_{h}$, where $R_{h}$ denotes the gradient recovery operator associated with SPR or PPR.

To recover the gradient using SPR or PPR at a mesh node $z=\left(x_{z}, y_{z}\right)$, a patch $\omega_{z}$ of triangles is selected as shown in Fig. 1(a). To recover the $x$-derivative at $z$ using SPR, we find a polynomial $p_{x} \in P_{1}\left(\omega_{z}\right)$ that best fits $\partial_{x} v_{h}$, in least-squares sense, at the triangles centers in $\omega_{z}$. The recovered $x$-derivative at $z$ is defined to be $p_{x}\left(x_{z}, y_{z}\right)$. Similarly, we can define the recovered $y$-derivative at $z$. To get the required polynomials, $\omega_{z}$ must have at least three triangles whose centers are not lying on one straight line. This requirement is guaranteed for $z \in \Omega$, but not for $z \in \partial \Omega$. For $z \in \partial \Omega$, the gradient is computed in every patch corresponding to an adjacent node in $\Omega$ by evaluating the obtained polynomials at $z$, and then taking the average. If there are no adjacent nodes in $\Omega$, then the gradient is defined to be $\nabla v_{h}(z)$ (see [13] for more details).

To recover the gradient at $z$ using PPR, we find a polynomial $p \in P_{2}\left(\omega_{z}\right)$ that best fits $v_{h}$, in least-squares sense, at the mesh nodes in $\omega_{z}$. The recovered gradient is defined to be $\nabla p\left(x_{z}, y_{z}\right)$. To get $p, \omega_{z}$ must contain at least 6 mesh nodes that are not on a conic section, as we shall see later. This might not be achieved if $\omega_{z}$ contains only the triangles attached to $z$. As it was proposed in [11], $\omega_{z}$ is extended by adding the triangles sharing an edge with $\omega_{z}$, as shown in Fig. 1(b). Nodes on $\partial \Omega$ are handled in the same way, although they need extra care in constructing their patches. Unlike SPR, PPR recovers the exact gradient if $v_{h} \in P_{2}\left(\omega_{z}\right)$ without any restrictions on $\mathcal{T}_{h}$.

After the previous description of PPR and SPR recovery techniques, we have the following important remarks.

Remark 1.1 It is easy to see that PPR preserves the first derivatives of polynomials in $P_{2}\left(\omega_{z}\right)$. This is not true for SPR, except for some special cases. Basically, PPR can be viewed as a dynamic way to generate difference schemes for first derivatives that can recover the exact derivatives of polynomials in $P_{2}\left(\omega_{z}\right)$. In [3], a technique was proposed to generate such kind of difference schemes a priori, as in example $4.8^{*} .4$, where the derivative at a mesh node $z$ is expressed as a weighted sum of the function values at the mesh nodes directly attached to $z$. The weights are determined such that

1. the first derivative derivatives of the basis of $P_{2}(\omega)$ are preserved, and 
2. the sum of the squares of the weights is minimum.

It can be shown that PPR generates weights satisfying the above conditions without worrying about the mesh structure, or doing that a priori as it is used in real time mode.

Remark 1.2 The idea of best fitting the function values by a quadratic polynomial was used before, as in [7] and [9], but it is used in a way that differs with the way it is used in PPR in many aspects.

1. It is mainly used for recovering the functions values, and not the derivatives.

2. The patches used in PPR are constructed for nodes, while in function recovery technique patches are constructed for triangles.

3. In PPR, the best fit quadratic polynomial is used to compute the gradient at the node for which the patch is constructed, while in function recovery technique the best fit polynomial is used to approximate the function on the triangle for which the patch was constructed.

As it was shown in [3], a posteriori error estimators based on function recovery techniques are inferior to many estimators, especially the one based on SPR.

Let $I_{h} u \in S_{h}$ be the Lagrange interpolation of $u$, and let $\omega_{T}=\bigcup\left\{\omega_{z}: z\right.$ is a vertex of $\left.T\right\}$ be a patch corresponding to $T \in \mathcal{T}_{h}$. If

$$
\left\|\nabla\left(I_{h} u-u_{h}\right)\right\|_{L^{2}\left(\omega_{T}\right)} \leq C h^{1+\rho}
$$

for some $\rho>0$, and

$$
\left\|R_{h} v\right\|_{L^{2}(T)} \leq C|v|_{1, \omega_{T}}
$$

for all $T \in \mathcal{T}_{h}$, and for all $v \in S_{h}$, then it is possible to show that

$$
\left\|\nabla u-R_{h} u_{h}\right\|_{L^{2}(\Omega)} \leq C h^{1+\rho},
$$

which means that the recovered gradient is superconvergent to the exact gradient. From that it is straight forward to prove that the a posteriori error estimator $\eta_{h}$ defined by

$$
\eta_{h}=\left\|R_{h} u_{h}-\nabla u_{h}\right\|_{L^{2}(\Omega)}
$$

is asymptotically exact. As it is usual in finite element analysis, $C$ is a generic constant that may depend on $u, \Omega$, or mesh parameters other than $h$.

The assumption in (1.6) is satisfied if $\mathcal{T}_{h}$ is mildly structured in the following sense. Let $\mathcal{T}_{h}=\mathcal{T}_{h, 1} \cup \mathcal{T}_{h, 2}$ and $\Omega_{h}=\Omega_{h, 1} \cup \Omega_{h, 2}$, where $\Omega_{h, i}=\bigcup_{T \in \mathcal{T}_{h, i}} T$ for $i=1,2$. 
Definition 1.3 The triangulation $\mathcal{T}_{h}$ is said to satisfy the condition $(\alpha, \sigma)$, if there exist positive constants $\alpha$ and $\sigma$ such that: Every two adjacent triangles in $\mathcal{T}_{h, 1}$ form an $O\left(h^{1+\alpha}\right)$ parallelogram, and $\left|\Omega_{h, 2}\right|=O\left(h^{\sigma}\right)$.

An $O\left(h^{\alpha+1}\right)$ parallelogram is a quadrilateral in which the difference between the lengths of any two opposite sides is $O\left(h^{\alpha+1}\right)$. When $\alpha=\infty$, then every pair of adjacent triangles in $\mathcal{T}_{h, 1}$ form a parallelogram. The case in which $\alpha=\sigma=\infty$ corresponds to $\mathcal{T}_{h}$ that is uniformly generated by lines parallel to three fixed directions. This case was handled in [6], where the error was expanded at mesh nodes, and the case in which $\alpha=1$ was handled in [5]. The general case was treated in [10], where the following theorem was established.

Theorem 1.4 Let $u$ be the solution of (1.4), let $u_{h} \in S_{h}$ be the finite element solution of (1.5), and let $I_{h} u \in S_{h}$ be the linear interpolation of $u$. If the triangulation $\mathcal{T}_{h}$ satisfies the condition $(\alpha, \sigma)$ and $u \in H^{3}(\Omega) \cap W_{\infty}^{2}(\Omega)$, then

$$
\left\|u_{h}-I_{h} u\right\|_{1, \Omega} \leq h^{1+\rho}\left(\|u\|_{3, \Omega}+|u|_{2, \infty, \Omega}\right),
$$

where $\rho=\min \left(\alpha, \frac{1}{2}, \frac{\sigma}{2}\right)$.

Remark 1.5 The condition $(\alpha, \sigma)$ is sufficient to guarantee the superconvergence result in (1.6), but it is not necessary, as we shall see later. But, this condition is satisfied for meshes generated by many automatic mesh generators, as described in [10],i.e. it covers a wide range of meshes.

The assumption in (1.7) requires the operator associated with the recovery technique to be bounded. This is somewhat easy to establish when the recovery technique works directly on $\nabla v$, which is the case in many recovery techniques like weighted average and SPR. In PPR the situation is much harder as it works on $v$. It is even not clear how to relate $R_{h} v$ to $\nabla v$.

From now on, $\mathrm{G}^{\mathrm{h}}: S_{h} \rightarrow S_{h} \times S_{h}$ denotes the operator associated with PPR. The main target in this paper is to show that $\mathrm{G}^{\mathrm{h}}$ satisfies the assumption in (1.7). Having this in hand paves the way to prove the superconvergence property in (1.8) when $R_{h} u_{h}=\mathrm{G}^{\mathrm{h}} u_{h}$. Finally, some numerical results are provided as a support for the theoretical results, where attention is paid to the regions near $\partial \Omega$. Also, the behavior of PPR is compared with that of SPR, as the later is widely used in practice.

Before going through the details, we should mention that PPR can be generalized to higher order elements, although it needs more care in selecting the sampling points. This will be handled in a future work. 


\section{Definition and existence of $\mathrm{G}^{\mathrm{h}}$}

Let $v \in S_{h}$. To define $\mathrm{G}^{\mathrm{h}} v \in S_{h} \times S_{h}$, it is enough to define it at each mesh node. Consider a mesh node $z=\left(x_{0}, y_{0}\right) \in \bar{\Omega}$, and its corresponding patch $\omega_{z}$ with triangles $T_{1}, T_{2}, \ldots, T_{m}$, and the nodes $z_{0}=z, z_{1}=\left(x_{1}, y_{1}\right), \ldots, z_{n}=\left(x_{n}, y_{n}\right)$. Without loss of generality, $z$ is considered as the origin of a local coordinate system for $\omega_{z}$, i.e. $z=(0,0)$; otherwise, we replace node $z_{i} \in \omega_{z}$ by $z_{i}-z$, for $i=0, \ldots, n$. Set $h_{z}=\max _{1 \leq i \leq n}\left\|z_{i}-z\right\|$. We may assume $h_{z}=1$, i.e. the whole patch is inside the unite circle centered at $z$; otherwise we replace node $z_{i} \in \omega_{z}$ by $z_{i} / h_{z}$ for $i=1, \ldots, n$. Let $v_{z, i}=v\left(z_{i}\right)$ for $i=0,1, \ldots, n$. Let $p_{z} \in P_{2}\left(\omega_{z}\right)$ be the quadratic polynomial that best fits $v$, in least-squares sense, on $\omega_{z}$. We write $p_{z}(x, y)=\boldsymbol{x}^{T} \boldsymbol{c}_{\boldsymbol{z}}$, where $(x, y) \in \omega_{z}$, $\boldsymbol{c}_{z}=\left[\begin{array}{llllll}c_{z, 1} & c_{z, 2} & c_{z, 3} & c_{z, 4} & c_{z, 5} & c_{z, 6}\end{array}\right]^{T}$, and $\boldsymbol{x}^{T}=\left[\begin{array}{llllll}1 & x & y & x^{2} & x y & y^{2}\end{array}\right]$. By definition, $\boldsymbol{c}_{z}$ is determined by the linear system $A^{T} A_{z} c_{z}=A^{T} \boldsymbol{v}_{z}$, where $\boldsymbol{v}_{z}=\left[\begin{array}{llll}v_{z, 0} & v_{z, 1} & \ldots & v_{z, n}\end{array}\right]^{T}$, and

$$
A_{z}=\left[\begin{array}{ccccc}
1 & x_{0} & y_{0} & \cdots & y_{0}^{2} \\
1 & x_{1} & y_{1} & \cdots & y_{1}^{2} \\
1 & x_{2} & y_{2} & \cdots & y_{2}^{2} \\
\vdots & \vdots & \vdots & \ddots & \vdots \\
1 & x_{n} & y_{n} & \cdots & y_{n}^{2}
\end{array}\right]
$$

Let $B_{z}=A_{z}^{T} A_{z}$, then, assuming the existence of $B_{z}^{-1}, c_{z}=B_{z}^{-1} A_{z}^{T} v_{z}$. By definition,

$$
\mathrm{G}^{\mathrm{h}} v(z)=\frac{\nabla p_{z}(0,0)}{h_{z}}=\frac{1}{h_{z}}\left[\begin{array}{cc}
c_{z, 2} & c_{z, 3},
\end{array}\right]^{T}=\frac{1}{h_{z}}\left[\begin{array}{ll}
v_{z}^{T} A_{z} B_{z}^{-1} e_{2} & v_{z}^{T} A_{z} B_{z}^{-1} e_{3}
\end{array}\right]^{T},
$$

where $e_{2}$ and $e_{3}$ are the second and the third columns of the identity matrix $I_{6 \times 6}$.

It is clear that computing $p_{z}$ requires at least 6 nodes, i.e., $n \geq 5$. Basically, the patch $\omega_{z}$ contains the triangles attached to $z$. If $n<5$, then $\omega_{z}$ is extended by attaching the triangles sharing an edge with it. For $z \in \Omega$, this extension is enough to get $n \geq 5$, but for $z \in \partial \Omega$ we may need to iterate this process more than once. Unfortunately having $n \geq 5$ is not enough to recover the gradient and other conditions have to imposed especially for nodes on the boundary. Before we continue, note that all quantities defined for $z$, or $\omega_{z}$, will be subscripted with $z$.

From the above discussion, it is important to answer the following questions, especially the second one:

1. Assuming existence of $p_{z}$, does it depend on the orientation of the local coordinate system at $z$ ? Also, does this orientation affect the accuracy of the numerical computations of $p_{z}$,i.e. the condition number of $A_{z}$ ?

2. Are there any sufficient conditions that guarantee the existence of $B_{z}^{-1}$ ? 
The following lemma addresses the answer of the first question.

Lemma 2.1 If $p_{z}$ exits, then it is invariant under the rotation of the local coordinate system at $z$.

Proof. Rotate the local coordinate system at $z$ by an angle $\theta$ in counterclockwise direction. The superscript ${ }^{\sim}$ will be used for quantities expressed in the rotated coordinate system. If $(x, y)$ refers to a point in the original coordinate system and $(\tilde{x}, \tilde{y})$ refers to the same point in the rotated coordinate system, then

$$
\left[\begin{array}{l}
x \\
y
\end{array}\right]=\left[\begin{array}{cc}
\cos \theta & -\sin \theta \\
\sin \theta & \cos \theta
\end{array}\right]\left[\begin{array}{l}
\widetilde{x} \\
\widetilde{y}
\end{array}\right]
$$

With this in mind, it is easy to verify that $\tilde{\boldsymbol{x}}^{T}=R_{\theta} \boldsymbol{x}^{T}$ and $\tilde{A}_{z}=A_{z} R_{\theta}$, where

$$
R_{\theta}=\left[\begin{array}{cccccc}
1 & 0 & 0 & 0 & 0 & 0 \\
0 & \cos \theta & -\sin \theta & 0 & 0 & 0 \\
0 & \sin \theta & \cos \theta & 0 & 0 & 0 \\
0 & 0 & 0 & \cos ^{2} \theta & -\cos \theta \sin \theta & \sin ^{2} \theta \\
0 & 0 & 0 & 2 \cos \theta \sin \theta & \cos ^{2} \theta-\sin { }^{2} \theta & -2 \cos \theta \sin \theta \\
0 & 0 & 0 & \sin ^{2} \theta & \cos \theta \sin \theta & \cos ^{2} \theta
\end{array}\right] .
$$

Also, it is easy to verify the following properties for $R_{\theta}$ :

1. $\operatorname{Det}\left(R_{\theta}\right)=1$, i.e., $R_{\theta}$ is invertible. Moreover, $R_{\theta}^{-1}=R_{-\theta}$.

2. The singular values of $R_{\theta}$ are $s_{\theta}^{-1}, 1,1,1,1$, and $s_{\theta}$, where $s_{\theta}=[9-\cos 4 \theta+((1-\cos 4 \theta)(17-$ $\left.\cos 4 \theta))^{1 / 2}\right]^{1 / 2} / 8$, and $1 \leq s_{\theta} \leq \sqrt{2}$. Note that $s_{-\theta}=s_{\theta}$, and so the singular values of $R_{-\theta}$ are the same as those of $R_{\theta}$. Hence, $\left\|R_{\theta}\right\|=\left\|R_{-\theta}\right\|=s_{\theta}$.

Let $\tilde{p}_{z}(\widetilde{x}, \widetilde{y})=\tilde{\boldsymbol{x}}^{T} \tilde{\boldsymbol{c}}_{z} \in P_{2}\left(\omega_{z}\right)$ be the least-squares best fit of $v$ on the same patch $\omega_{z}$, but with respect to rotated coordinate system at $z$. As before, $\tilde{c}_{z}$ is determined by solving the linear system $\tilde{A}_{z}{ }^{T} \tilde{A}_{z} \tilde{c}_{z}={\tilde{A_{z}}}^{T} \boldsymbol{v}_{z}$. Since $\tilde{B}_{z}={\tilde{A_{z}}}^{T} \tilde{A}_{z}=R_{\theta}^{T} B_{z} R_{\theta}$, and $B_{z}^{-1}$ is assumed to exist, then $\tilde{B}_{z}^{-1}=R_{\theta}^{-1} B_{z}^{-1} R_{\theta}^{-T}$. Hence, $\tilde{c}_{z}=\tilde{B}_{z}^{-1} \tilde{A}_{z}^{T} v_{z}=R_{\theta}^{-1} c_{z}$, i.e., $\tilde{p}_{z}=\tilde{\boldsymbol{x}}^{T} R_{\theta}^{-1} c_{z}=x c_{z}=p_{z}$, and the proof is complete.

For any Matrix $H$ of order $k_{1} \times k_{2}$, let $\sigma_{1}(H)$, and $\sigma_{\min (k 1, k 2)}(H)$ denote the largest and the smallest singular values of $H$, respectively. As we know, $\sigma_{l}^{2}(H)=\sigma_{l}\left(H^{T} H\right)$, for $l=$ $1,2, \ldots, \min (k 1, k 2)$.

Remark 2.2 From the proof of Lemma 2.1, we know that $\tilde{B}_{z}=R_{\theta}^{T} B_{z} R_{\theta}$. Using this relation, the properties of singular values, and the properties of $R_{\theta}$, it is easy to verify that

$$
\frac{\sigma_{l}\left(A_{z}\right)}{\sqrt{2}} \leq s_{\theta}^{-1} \sigma_{l}\left(A_{z}\right) \leq \sigma_{l}\left(\tilde{A}_{z}\right) \leq s_{\theta} \sigma_{l}\left(A_{z}\right) \leq \sqrt{2} \sigma_{l}\left(A_{z}\right) \text { for } l=1,6
$$

This shows that the patch orientation has almost no effect on the condition number of $A_{z}$. 
We turn our attention now to the answer of the second question. We start with the following theorem from [8], after adopting our notation.

Theorem 2.3 Pointwise interpolation in $P_{2}(\mathcal{A}), \mathcal{A} \subseteq \mathbb{R}^{2}$, at six distinct points $\left(x_{i}, y_{i}\right) \in \mathcal{A}, i=$ $1,2, \ldots, 6$, has the finite interpolation property if and only if there is no conic section passing through all of six points.

The above theorem simply says that the interpolation by a quadratic polynomial at six nodes exists and is unique as long as the six points are not on a conic section.

Definition 2.4 The patch $\omega_{z}$ is said to satisfy the angle condition if the sum of any two adjacent angles inside $\omega_{z}$ is at most $\pi$, and is said to satisfy the line condition if its nodes are not lying on two lines.

We write $n=n_{1}+n_{2}$, where $n_{1}$ denotes the number of nodes that are directly attached to $z$. If $z \in \Omega$, then $n_{1} \geq 3$. Practically, a good mesh generator can detect any node $z$ for which $n_{1}=3$ and removes it. So, we may assume that $n_{1} \geq 4$. It is obvious that for $z \in \Omega$ with $n_{1}>4, \omega_{z}$ satisfies the line condition, unless one of its triangles is degenerate. If $n_{1}=4, \omega_{z}$ may violate this condition as shown in Fig. 3(a). The following elementary lemma is needed in the proof of Theorem 2.6.

Lemma 2.5 Any tangent to a branch of a hyperbola can not intersect with the other branch.

Theorem 2.6 Let $z \in \Omega$ be a mesh node. If the patch $\omega_{z}$ corresponding to $z$ satisfies the angle and the line conditions, then $B_{z}$ is invertible.

Proof. As we know, if $\operatorname{Rank} A_{z}=6$, then $\operatorname{Rank} B_{z}=6$, and $B_{z}$ is invertible. By Theorem 2.3, it is enough to show that $\omega_{z}$ has six distinct nodes that are not on a conic section. Having $z \in \Omega$ implies that sum of the angles at $z_{0}$ is $2 \pi$. Hence, the nodes in $\omega_{z}$ can not lie on a circle, a parabola, an ellipse, or on one branch of a hyperbola. Since $\omega_{z}$ satisfies the line condition, the nodes can not be on two lines. The remaining possibility is to have the nodes distributed on two branches of a hyperbola. Depending on $n_{1}$, we can have one of the following two cases.

Case 1: $n_{1}>4$. Proceed by contradiction, and assume that the nodes in $\omega_{z}$ are distributed on two branches of a hyperbola. Without loss of generality, one may assume that the real axis of the hyperbola is horizontal, and $z_{0}$ lies on the right branch. The left branch must have at least one node of $\omega_{z}$. If it has three nodes, as in Fig. 2(a), then the angle condition is violated as the measure of the angle $z_{1} z_{2} z_{3}>\pi$. Using the same argument, the left branch can not have 
more than two nodes. If it has two nodes, as shown in Fig. 2(b), then $\omega_{z}$ must have nodes $z_{3}$ and $z_{4}$ connected to $z_{1}$ and $z_{2}$, respectively. To satisfy the angle condition, $\omega_{z}$ can not have any nodes, like $z_{5}$, along the hyperbola to the right of $z_{3}$. This is because the line $z_{1} z_{3}$ can not be tangent to the hyperbola at $z_{3}$ by Lemma 2.5. Using the same argument, we can not have any nodes, like $z_{6}$, along the hyperbola to the right of $z_{4}$. Thus, to comply with the angle condition, $\omega_{z}$ must have $n_{1}=4$, and this is a contradiction as $n_{1}>4$. Finally, if the left branch has just one node of $\omega_{z}$, as in Fig. 2(c), then $\omega_{z}$ must have nodes $z_{2}$ and $z_{3}$ connected to $z_{1}$. Again, to satisfy the angle condition, $n_{1}=3$, and this is a contradiction.

Case 2: $n_{1}=4$. In this case the triangles attached to $z_{0}$ forms a quadrilateral. Let $z_{1}, z_{2}, z_{3}$, and $z_{4}$ be the vertices of this quadrilateral taken in a counterclockwise direction, as shown in Fig. 3(a). Since $\omega_{z}$ satisfies the angle condition, $z_{0}$ must be the intersection point of the quadrilateral diagonals. The nodes in $\omega_{z}$ can not be distributed on a hyperbola; otherwise each diagonal intersects with the hyperbola at three points, which is impossible, and this completes the proof.

The situation for nodes on $\partial \Omega$ is more delicate. Angle and line conditions are not sufficient any more, as shown in Fig. 3(b). So, in constructing $\omega_{z}$ for $z \in \partial \Omega$ we must impose another condition that ensures the invertibility of $B_{z}$. From what has been established, this condition is obvious and is a direct corollary of Theorem 2.6 .

Corollary 2.7 Consider a mesh node $z \in \partial \Omega$, and let $\omega_{z}$ be its corresponding patch. Suppose that $\omega_{z}$ contains another patch $\omega_{\hat{z}}$ corresponding to a node $\hat{z} \in \Omega \cap \omega_{z}$ and $\omega_{\hat{z}}$ satisfies the angle and line conditions. Then, $B_{z}$ is invertible.

Hence in constructing $\omega_{z}$ for $z \in \partial \Omega, \omega_{z}$ is extended, as described before, till it contains a patch corresponding to a node in $\Omega$. The time-cost for this extension might be expensive. A cheaper procedure is to construct $\omega_{z}$ such that it contains all the nodes up to the nearest nodes in $\Omega$ and their corresponding patches. In this case the number of nodes is larger, and the computational cost might get higher.

\section{Boundedness of $\mathrm{G}^{\mathrm{h}}$}

Before we start investigating the boundedness of $\mathrm{G}^{\text {h }}$, we go over some basic facts. Consider a mesh triangle $T \subset \bar{\Omega}$ with vertices $\left(x_{1}, y_{1}\right),\left(x_{2}, y_{2}\right)$, and $\left(x_{3}, y_{3}\right)$ taken in counterclockwise direction. If the nodal value of $v \in S_{h}$ at $\left(x_{j}, y_{j}\right)$ is $v_{j}$, and the basis function associated with this node is $\theta_{j}$, then, for $(x, y) \in T, v(x, y)=\sum_{j=1}^{3} v_{j} \theta_{j}(x, y)$. Consider the reference triangle $\hat{T}$ with the vertices $\left(\xi_{1}, \eta_{1}\right)=(0,0),\left(\xi_{2}, \eta_{2}\right)=(1,0)$, and $\left(\xi_{3}, \eta_{3}\right)=(1,0)$. The basis functions 
on $\hat{T}$ are $\lambda_{1}(\xi, \eta)=1-\xi-\eta, \lambda_{2}(\xi, \eta)=\xi$, and $\lambda_{3}(\xi, \eta)=\eta$, where $\lambda_{j}$ is associated with the vertex $\left(\xi_{j}, \eta_{j}\right), j=1,2,3$. Let $F: \hat{T} \rightarrow T$ be the transformation defined by

$$
F(\xi, \eta)=\left[\begin{array}{l}
x(\xi, \eta) \\
y(\xi, \eta)
\end{array}\right]
$$

where

$$
x(\xi, \eta)=\sum_{j=1}^{3} x_{j} \lambda_{j}=x_{1}+\left(x_{2}-x_{1}\right) \xi+\left(x_{3}-x_{1}\right) \eta
$$

and

$$
y(\xi, \eta)=\sum_{j=1}^{3} y_{j} \lambda_{j}=y_{1}+\left(y_{2}-y_{1}\right) \xi+\left(y_{3}-y_{1}\right) \eta .
$$

The jacobian of this transformation is

$$
J=\left[\begin{array}{cc}
x_{2}-x_{1} & x_{3}-x_{1} \\
y_{2}-y_{1} & y_{3}-y_{1}
\end{array}\right]
$$

As we know, $\lambda_{j}=\theta_{j} \circ F$ for $j=1,2,3$. If $\hat{v}=v \circ F$, then

$$
\hat{v}(\xi, \eta)=v(x(\xi, \eta), y(\xi, \eta))=\sum_{j=1}^{3} v_{j} \lambda_{j}(\xi, \eta)
$$

Writing the gradient as a column vector, we get

$$
\nabla v=\frac{1}{h_{z}} J^{-T} \nabla \hat{v}
$$

Since

$$
\nabla \hat{v}=\left[\begin{array}{lll}
-1 & 1 & 0 \\
-1 & 0 & 1
\end{array}\right]\left[\begin{array}{l}
v_{1} \\
v_{2} \\
v_{3}
\end{array}\right]
$$

then

$$
\nabla v=\frac{1}{2|T|}\left[\begin{array}{ccc}
y_{2}-y_{3} & y_{3}-y_{1} & y_{1}-y_{2} \\
x_{3}-x_{2} & x_{1}-x_{3} & x_{2}-x_{1}
\end{array}\right]\left[\begin{array}{c}
v_{1} \\
v_{2} \\
v_{3}
\end{array}\right]
$$

Setting

$$
a_{j}=\frac{1}{2|T|}\left(y_{j+1}-y_{j+2}\right), \text { and } b_{j}=\frac{1}{2|T|}\left(x_{j+2}-x_{j+1}\right),
$$

where the addition in indices is $\bmod 3$, we have, for $(x, y) \in T$,

$$
\partial_{x} v(x, y)=\sum_{j=1}^{3} a_{j} v_{j} \text { and } \partial_{y} v(x, y)=\sum_{j=1}^{3} b_{j} v_{j}
$$


Let $\omega_{z}$ be the patch corresponding to $z \in \bar{\Omega}$, and consider a triangle $T_{k} \subset \omega_{z}$ for some $1 \leq k \leq m$. If the vertices of $T_{k}$, taken in counterclockwise direction, are $\left(x_{k, 1}, y_{k, 1}\right),\left(x_{k, 2}, y_{k, 2}\right)$, and $\left(x_{k, 3}, y_{k, 3}\right)$, then for $(x, y) \in T_{k}$, and using (3.1) and (3.2),

$$
\partial_{x} v(x, y)=\frac{1}{h_{z}} \sum_{j=1}^{3} a_{k, j} v_{k, j}, \text { and } \partial_{y} v(x, y)=\frac{1}{h_{z}} \sum_{j=1}^{3} b_{k, j} v_{k, j},
$$

where

$$
a_{k, j}=\frac{1}{2\left|T_{k}\right|}\left(y_{k, j+1}-y_{k, j+2}\right), \text { and } b_{k, j}=\frac{1}{2\left|T_{k}\right|}\left(x_{k, j+2}-x_{k, j+1}\right),
$$

and $v_{k, j}=v\left(x_{k, j}, y_{k, j}\right)$. Hence,

$$
\partial_{x} v(x, y)=\frac{1}{h_{z}} \boldsymbol{v}_{k}^{T} \boldsymbol{a}_{k}, \text { and } \partial_{y} v(x, y)=\frac{1}{h_{z}} \boldsymbol{v}_{k}^{T} \boldsymbol{b}_{k},
$$

where $\boldsymbol{a}_{k}=\left[\begin{array}{lll}a_{k, 1} & a_{k, 2} & a_{k, 3}\end{array}\right]^{T}, \boldsymbol{b}_{k}=\left[\begin{array}{lll}b_{k, 1} & b_{k, 2} & b_{k, 3}\end{array}\right]^{T}$, and $\boldsymbol{v}_{k}=\left[\begin{array}{lll}v_{k, 1} & v_{k, 2} & v_{k, 3}\end{array}\right]^{T}$. Let $E_{k}$ be an $(n+1) \times 3$ Boolean matrix defined for $T_{k}$, where

$$
E_{k}(i, j)=\left\{\begin{array}{ll}
1 & \text { if the node } i \text { in } \omega_{z} \text { is the vertex } j \text { in } T_{k} \\
0 & \text { otherwise }
\end{array},\right.
$$

then $v_{k}=E_{k}^{T} v_{z}$, and

$$
\partial_{x} v(x, y)=\frac{1}{h_{z}} v_{z}^{T} E_{k} a_{k}, \text { and } \partial_{y} v(x, y)=\frac{1}{h_{z}} v_{z}^{T} E_{k} b_{k} .
$$

Let $\mathrm{G}_{1}{ }_{1} v$ and $\mathrm{G}_{2}{ }_{2} v$ stand for the recovered $x-$ and $y$-derivatives, respectively. Establishing the boundedness of $\mathrm{G}^{\mathrm{h}}$ in the sense of $(1.7)$ would be easy if $\mathrm{G}_{l}^{\mathrm{h}} v(z)$ can be expressed as a liner combination of the first derivatives of $v$ on the triangles of $\omega_{z}$, for $l=1,2$. So, we will try to find a set of bounded values $\alpha_{z, l, 1}, \ldots, \alpha_{z, l, m}$, and $\beta_{z, l, 1}, \ldots, \beta_{z, l, m}$ such that

$$
\mathrm{G}_{l}^{\mathrm{h}} v(z)=\sum_{k=1}^{m}\left[\beta_{z, l, k}\left(\partial_{x} v\right)_{k}+\alpha_{z, l, k}\left(\partial_{y} v\right)_{k}\right], \quad l=1,2
$$

where $\left(\partial_{x} v\right)_{k}$ and $\left(\partial_{y} v\right)_{k}$ are the derivatives of $v$ on triangle $T_{k}$. Using equations (2.2) and (3.3) we have

$$
\frac{1}{h_{z}} \boldsymbol{v}_{z}^{T} \sum_{k=1}^{m}\left[\beta_{z, l, k} E_{k} \boldsymbol{a}_{k}+\alpha_{z, l, k} E_{k} \boldsymbol{b}_{k}\right]=\frac{1}{h_{z}} \boldsymbol{v}_{z}^{T} A_{z} B_{z}^{-1} e_{l+1}, \quad l=1,2
$$

Setting

$$
M_{z}=\left[\begin{array}{llllll}
E_{1} a_{1} & \cdots & E_{m} a_{m} & E_{1} b_{1} & \cdots & E_{m} b_{m}
\end{array}\right]
$$

and

$$
\gamma_{z, l}=\left[\begin{array}{llllll}
\alpha_{z, l, 1} & \cdots & \alpha_{z, l, m} & \beta_{z, l, 1} & \cdots & \beta_{z, l, m}
\end{array}\right]^{T}
$$


we get

$$
\frac{1}{h_{z}} v_{z}^{T} M_{z} \gamma_{z, l}=\frac{1}{h_{z}} v_{z}^{T} A_{z} B_{z}^{-1} e_{l+1}, \quad l=1,2 .
$$

This is true for all $v \in S_{h}$, and so

$$
M_{z} \gamma_{z, l}=A_{z} B_{z}^{-1} e_{l+1} ; \quad l=1,2
$$

Note that the order of $M_{z}$ is $(n+1) \times(2 m)$.

Lemma 3.1 Consider a mesh node $z \in \bar{\Omega}$. If the patch $\omega_{z}$ corresponding to $z$ has no degenerate triangles and $B_{z}$ is invertible, then $\operatorname{Rank} M_{z}=n$, and the system in (3.6) has infinitely many solutions.

Proof. Since $\omega_{z}$ is simply connected, and using Euler's theorem, $(n+1)-e+m=1$, where $e$ is the number of edges in $\omega_{z}$. Hence, $(n+1)-2 m=e-3 m+1$. By a simple induction argument on $m$, we can show that $e-3 m+1<0$ for $m \geq 3$. Hence, the system in (3.6) is underdetermined.

To prove that $\operatorname{Rank} M_{z}=n$, we consider the homogeneous linear system

$$
M_{z}^{T} w=0
$$

with $w=\left[\begin{array}{llll}w_{0} & w_{1} & \cdots & w_{n}\end{array}\right]^{T}$. We can view $w_{0}, w_{1}, \ldots, w_{n}$ as the nodal values of some function $w \in S_{h}$ at the nodes of $\omega_{z}$. With this in mind, equations $j$ and $j+m$ of the homogeneous system in (3.7) leads to $\nabla w=0$ on $T_{k}$, for $k=1,2, \ldots, m$, and so $w$ must be constant on $\omega_{z}$. Since $w$ is piecewise linear, the only solution to this homogeneous system is $w_{0}=w_{1}=\ldots=w_{n}$. Therefore, the dimension of the null space of $M_{z}^{T}$ is 1 , and $\operatorname{Rank} M_{z}^{T}=\operatorname{Rank} M_{z}=n$. Also, this implies that the only row operation on $M_{z}$ that leads to a row of zeros is adding all the rows together. Since $G^{\text {h }}$ recovers the exact gradient for any polynomial $p \in P_{2}\left(\omega_{z}\right)$, it is easy to verify that the sum of the rows of the column $A_{z} B_{z}^{-1} e_{l+1}=0$ for $l=1,2$, and so the homogeneous system in (3.6) is consistent for $l=1,2$.

Among all the solutions of (3.6), we consider the one with the minimum length given by

$$
\gamma_{z, l}^{*}=M_{z}^{\dagger} A_{z} B_{z, l+1}^{-1}, \quad l=1,2
$$

where $M_{z}^{\dagger}$ is the pseudoinverse of $M_{z}$. As before, let $\omega_{T}=\bigcup\left\{\omega_{z}: z\right.$ is a vertex of $\left.T\right\}$ be a patch corresponding to triangle $T \in \bar{\Omega}$. 
Theorem 3.2 Let $0<C_{1} \leq \sigma_{6}\left(A_{z}\right) \leq \sigma_{1}\left(A_{z}\right) \leq C_{2}$ and $0<C_{3} \leq \sigma_{n}\left(M_{z}\right)$ for every mesh node $z \in \bar{\Omega}$ and for some constants $C_{1}, C_{2}$ and $C_{3}$ that are independent of $h$. Then there exist a constant $C$, independent of $h$, such that

$$
\left\|\mathrm{G}^{\mathrm{h}} v\right\|_{L^{2}(T)} \leq C|v|_{1, \omega_{T}}
$$

for all $T \subset \bar{\Omega}$, and for all $v \in S_{h}$

Proof. Consider a mesh triangle $T \subset \bar{\Omega}$, and let $z$ be one of its vertices. Let $\omega_{T}$ be the patch corresponding to $T$, and let $v$ be any function in $S_{h}$. Using equations (3.4) and (3.8) we get

$$
\begin{aligned}
\left|\mathrm{G}_{l}^{\mathrm{h}} v(z)\right| & \leq\left\|\gamma_{l}^{*}\right\|_{1}|v|_{1, \infty, \omega_{z}} \leq c_{1}\left\|\gamma_{l}^{*}\right\|_{2}|v|_{1, \infty, \omega_{T}} \\
& \leq c_{2}\left\|M^{\dagger}\right\|_{2}\|A\|_{2}\left\|B^{-1}\right\|_{2}|v|_{1, \infty, \omega_{T}} \\
& \leq \frac{c_{2} C_{2}}{C_{3} C_{1}^{2}}|v|_{1, \infty, \omega_{T}}
\end{aligned}
$$

for $l=1,2$. By linearity of $\mathrm{G}^{\mathrm{h}} v$ on $T$, we have

$$
\left\|\mathrm{G}^{\mathrm{h}} v\right\|_{L^{\infty}(T)} \leq C|v|_{1, \infty, \omega_{T}}
$$

Hence,

$$
\begin{aligned}
\left\|\mathrm{G}^{\mathrm{h}} v\right\|_{L^{2}(T)} & \leq \sqrt{|T|}\left\|\mathrm{G}^{\mathrm{h}} v\right\|_{L^{\infty}(T)} \leq C \operatorname{diam}(T)|v|_{1, \infty, \omega_{T}} \\
& \leq C \frac{\operatorname{diam}(T)}{\operatorname{diam}\left(\omega_{T}\right)}|v|_{1, \omega_{T}} \leq C|v|_{1, \omega_{T}} .
\end{aligned}
$$

The first inequality in (3.10) is obtained using an inverse estimate.

It is obvious that the bounds assumed about the singular values of $A_{z}$ and $M_{z}$ in Theorem 3.2 are mesh dependent. To simplify the situation, consider the unit disc

$$
\overline{B(0,1)}=\left\{(x, y) \in \mathbb{R}^{2}: x^{2}+y^{2} \leq 1\right\} \text {. }
$$

Let $\mathcal{T}$ be a triangulation of $\overline{B(0,1)}$ that is similar to one of the patterns shown in Fig. 4. Let $z_{0}=(0,0), z_{1}, \ldots, z_{n}$ be the nodes of $\mathcal{T}$ with at least one of them on the unit circle. Let $T_{1}, T_{2}, \ldots, T_{n}$ be the triangles of $\mathcal{T}$ with $\theta_{m}$ and $\theta_{M}$ being the smallest and the largest angles in any one of these triangles. As before, write $n=n_{1}+n_{2}$ with $n_{1}$ denoting the number of nodes directly linked to $z_{0}$. If $n_{1}=4$, let $D$ be the set of the diagonals of the quadrilateral $z_{1} z_{2} z_{3} z_{4}$, and set $d=\min _{5 \leq i \leq n} \operatorname{dist}\left(z_{i}, D\right)$. Let $A$ and $M$ be the matrices defined for $\omega$ as in equations (2.1) and (3.5), respectively, and set $B=A^{T} A$. 
Lemma 3.3 Let $\mathcal{T}$ be any triangulation of $\overline{B(0,1)}$ that is similar to one of the patterns shown in Fig. 4. Assume that $\mathcal{T}$ satisfies the following conditions for some positive constants $N, \delta, \phi_{m}$, and $\phi_{M}$ :

1. $\omega$ satisfies the angle condition,

2. $n_{1}<N<\infty$,

3. $0<\delta \leq d$, and

4. $0<\phi_{m} \leq \theta_{m}$, and $\theta_{M} \leq \phi_{M}<\pi$,

Then, there exist constants $C_{1}, C_{2}$, and $C_{3}$, that depend only on $N, \delta, \phi_{m}$, and $\phi_{M}$, such that

$$
0<C_{1} \leq \sigma_{6}(A) \leq \sigma_{1}(A) \leq C_{2}, \quad \text { and } \quad 0<C_{3} \leq \sigma_{n}(M)
$$

Proof. Note that the condition about $d$ is to ensure that $\omega$ satisfies the line condition when $n_{1}=4$. We now show that $\sigma_{1}(A) \leq C_{2}$. Using the definition of $A$, it is easy to verify that $|B(i, j)| \leq(n+1)$ for $1 \leq i, j \leq 6$. Hence, $\sigma_{1}(A)=\sqrt{\sigma_{1}(B)} \leq \sqrt{\sigma_{1}(|B|)} \leq \sqrt{6(n+1)} \leq$ $\sqrt{6(N+5)}=C_{2}$. To show that $0<C_{1} \leq \sigma_{6}(A)$ proceed by contradiction. Note that $\overline{B(0,1)}$ and $\left[\phi_{m}, \phi_{M}\right]$ are compact, and $\omega$ has at least one of its nodes on $\partial B(0,1)$. Based on that, a standard argument can be used to establish the existence of a patch $\omega$ that satisfies the given conditions and for which $\mathrm{B}$ is singular, i.e. $\sigma_{6}(A)=0$. Since $\omega$ satisfies the angle and the line conditions, $B$ is nonsingular by Theorem 2.6, and this is a contradiction. Similarly, we can show that $0<C_{3} \leq \sigma_{n}(M)$. Note that, under the given conditions, none of the given triangles in $\omega$ is degenerate and so $\operatorname{Rank} M=n$ by Lemma 3.1, i.e. $\sigma_{n}(M)$ can not be zero.

Theorem 3.4 Let $\mathcal{T}_{h}$ be a triangulation of $\Omega$ that satisfy the following conditions for any $h$.

1. If $n_{1}$ is the number of nodes directly attached to a mesh node $z$, then $4 \leq n_{1} \leq N$ for $z \in \Omega$, and $1 \leq n_{1} \leq N$ for $z \in \partial \Omega$, where $N$ is some finite positive integer.

2. If $n_{1}=4$ for a mesh node $z \in \Omega$, then the sum of any two adjacent angles at $z$ is $\pi$.

3. If $n_{1}>4$ for a mesh node $z \in \Omega$, then the sum of any two adjacent angles at $z$ is at most $\pi-\phi$ for some $\phi>0$.

4. If $z \in \partial \Omega$, then the sum of any two adjacent angles at $z$ is at most $\pi$. 
5. If $\theta_{m, h}$ and $\theta_{M, h}$ are the smallest and largest angles in any mesh $T \in \mathcal{T}_{h}$, then

$$
0<\phi_{m} \leq \theta_{m, h} \leq \theta_{M, h} \leq \phi_{m}<\pi
$$

for some constants $0<\phi_{m} \leq \phi_{M}<\pi$.

Then, there exist constants $C_{1}, C_{2}$, and $C_{3}$, that depend only on $N, \phi, \phi_{m}$, and $\phi_{M}$, such that

$$
0<C_{1} \leq \sigma_{6}\left(A_{z}\right) \leq \sigma_{1}\left(A_{z}\right) \leq C_{2}, \quad \text { and } \quad 0<C_{3} \leq \sigma_{n}\left(M_{z}\right)
$$

for all $z \in \Omega$

Proof. Let $z \in \Omega$, and let $\omega_{z}$ be its corresponding patch. Let $\omega=\frac{\omega_{z}-z}{h_{z}}$. The given conditions implies that $\omega$ satisfies the conditions in Lemma 3.3, and the theorem conclusion is true for all $z \in \Omega$.

Remark 3.5 Examining the assumptions in Theorem 3.4, we can see that the important ones are the those about the angles. The second assumption is easy to achieve during mesh generation. As was shown in [2], it is desirable to avoid having large angles, and so the assumptions from 3 to 5 seems to be practical.

Lemma 3.6 Let $\mathcal{T}_{h}$ be a triangulation of $\Omega$ that satisfies the assumptions in Theorem 3.4 for any $h$. Also assume that for any mesh node $z \in \partial \Omega$ the following conditions are satisfied:

1. $z$ is connected to at least one mesh node $\hat{z} \in \Omega$ directly or through a node $\tilde{z} \in \partial \Omega$, and

2. $\omega_{z}$ is constructed such that it contains a patch $\omega_{\hat{z}}$ corresponding to a mesh node $\hat{z} \in \Omega \cap \omega_{\hat{z}}$.

Then, there exist constants $C_{1}, C_{2}$, and $C_{3}$, independent of $h$, such that

$$
0<C_{1} \leq \sigma_{6}\left(A_{z}\right) \leq \sigma_{1}\left(A_{z}\right) \leq C_{2}, \quad \text { and } \quad 0<C_{3} \leq \sigma_{n}\left(M_{z}\right)
$$

for all $z \in \partial \Omega$.

Proof. Let $z \in \partial \Omega$, and let $\omega_{z}$ be its corresponding patch that satisfies the second assumption. Let $\omega=\frac{\omega_{z}-z}{h_{z}}$, and let $\omega_{1}=\frac{\omega_{\hat{z}}-z}{h_{z}}$. By the first assumption, the number of nodes in $\omega$ is bounded by $1+N^{2}$ when $z$ is directly attached to $\hat{z}$. So, if $z$ is connected to $\hat{z} \in \Omega$ through $\tilde{z} \in \partial \Omega$ then, the number of nodes in $\omega$ is at most $1+N\left(1+N^{2}\right)$. Using an argument similar to that used in Lemma 3.3, it is easy to find the constant $C_{2}$. To prove the existence of $C_{1}$, proceed by contradiction. Again, based on the compactness of $\overline{B(0,1)}$ and $\left[\phi_{m}, \phi_{M}\right]$, a standard argument 
can be used to establish the existence of a patch $\omega$ for which B is singular, and $\omega \supset \omega_{1}$ with $\omega_{1}$ satisfying the angle and the line conditions, by the assumptions in Theorem 3.4. we can write $A=\left[A_{1}^{T} A_{2}^{T}\right]^{T}$, where $A_{1}$ corresponds to $\omega_{1}$. Then, $B=A^{T} A=A_{1}^{T} A_{1}+A_{2}^{T} A_{2}=B_{1}+B_{2}$, where $B_{1}=A_{1}^{T} A_{1}$ and $B_{2}=A_{2}^{T} A_{2}$. Note that $B$ is positive semidefinite, as $B$ is singular, and $B_{2}$ is at least positive semidefinite. So, we can find a vector $c \in \mathbb{R}^{6}$ such that $c^{T} B c=c^{T} B_{1} c+c^{T} B_{2} c=0$, and, as a result, $c^{T} B_{1} c=0$. This means that $B_{1}$ is singular, and this is a contradiction as $\omega_{1}$ satisfies the angle and the line conditions. Similarly, we can establish the existence of $C_{3}$.

Remark 3.7 The first assumption in Lemma 3.6 can be relaxed, and in this case $C_{2}$ might get lager as the number of nodes in patches corresponding to nodes on $\partial \Omega$ will increase, and $C_{1}$ might get smaller as the $h_{\hat{z}} / h_{z}$ will decrease.

From Theorem 3.4 and Lemma 3.6, we have the following corollary.

Corollary 3.8 Let $\mathcal{T}_{h}$ be a triangulation of $\Omega$ that satisfies the conditions in Theorem 3.4 for any $h$, then there exist constants $C_{1}, C_{2}$, and $C_{3}$, independent of $h$, such that

$$
0<C_{1} \leq \sigma_{6}\left(A_{z}\right) \leq \sigma_{1}\left(A_{z}\right) \leq C_{2}, \quad \text { and } \quad 0<C_{3} \leq \sigma_{n}\left(M_{z}\right)
$$

for all $z \in \bar{\Omega}$.

\section{Superconvergence Property of PPR-Recovered Gradient}

We begin with the following main theorem.

Theorem 4.1 Let $\mathcal{T}_{h}$ be a triangulation of $\Omega$ that satisfies the condition $(\alpha, \sigma)$, and the assumptions in both of Theorem 3.4 and Lemma 3.6. If $u \in W_{\infty}^{3}(\Omega)$, then

$$
\left\|\nabla u-\mathrm{G}^{\mathrm{h}} u_{h}\right\|_{L^{2}(\Omega)} \leq C h^{1+\rho}\|u\|_{3, \infty, \Omega},
$$

where $\rho=\min \left(\alpha, \frac{1}{2}, \frac{\sigma}{2}\right)$.

Proof. Under the given assumptions, we have

$$
\left\|\mathrm{G}^{\mathrm{h}} v\right\|_{L^{2}(T)}<C_{1}\|\nabla v\|_{L^{2}\left(\omega_{T}\right)} \text { for all } v \in S_{h} \text { and } T \in \mathcal{T}_{h}
$$

for some $C_{1}$ that is independent of $h$, and

$$
\left\|\nabla\left(I_{h} u-u_{h}\right)\right\|_{L^{2}(\Omega)} \leq C_{2} h^{1+\rho}\|u\|_{3, \infty, \Omega}
$$


where $\rho=\min \left(\alpha, \frac{1}{2}, \frac{\sigma}{2}\right)$. We write

$$
\nabla u-\mathrm{G}^{\mathrm{h}} u_{h}=\left(\nabla u-\mathrm{G}^{\mathrm{h}}\left(I_{h} u\right)\right)+\left(\mathrm{G}^{\mathrm{h}}\left(I_{h} u-u_{h}\right)\right)
$$

As it was shown in [11],

$$
\left\|\nabla u-\mathrm{G}^{\mathrm{h}}\left(I_{h} u\right)\right\|_{L^{\infty}(\Omega)} \leq C h^{2}|u|_{3, \infty, \Omega} .
$$

Hence,

$$
\left\|\nabla u-\mathrm{G}^{\mathrm{h}}\left(I_{h} u\right)\right\|_{L^{2}(\Omega)} \leq C h^{2} \sqrt{|\Omega|}\|u\|_{3, \infty, \Omega} .
$$

For the second part in equation (4.3), and by virtue of (4.1), we have

$$
\begin{aligned}
\left\|\mathrm{G}^{\mathrm{h}}\left(I_{h} u-u_{h}\right)\right\|_{L^{2}(\Omega)}^{2} & =\sum_{T \in \mathcal{T}_{h}}\left\|\mathrm{G}^{\mathrm{h}}\left(I_{h} u-u_{h}\right)\right\|_{L^{2}(T)}^{2} \\
& \leq \sum_{T \in \mathcal{T}_{h}} C_{1}^{2}\left\|\nabla\left(I_{h} u-u_{h}\right)\right\|_{L^{2}\left(\omega_{T}\right)}^{2} \\
& \leq C\left\|\nabla\left(I_{h} u-u_{h}\right)\right\|_{L^{2}(\Omega)}^{2} .
\end{aligned}
$$

Consequently, and by using (4.2),

$$
\left\|\mathrm{G}^{\mathrm{h}}\left(I_{h} u-u_{h}\right)\right\|_{L^{2}(\Omega)} \leq C h^{1+\rho}\|u\|_{3, \infty, \Omega} .
$$

Using (4.5) and (4.6) in (4.3), we have the required result.

Remark 4.2 We should mention that the conclusion of Theorem 4.1 is true under any conditions that guarantee the results in (4.1) and (4.2). In other words, $\mathrm{G}^{\mathrm{h}}$, provided that it is bounded, can sense any superconvergence in $\nabla\left(I_{h} u-u_{h}\right)$, and produces a superconvergent recovered gradient.

Consider the global a posteriori error estimator $\eta_{h}$ defined by

$$
\eta_{h}=\left\|\mathrm{G}^{\mathrm{h}} u_{h}-\nabla u_{h}\right\|_{L^{2}(\Omega)} .
$$

Under the assumptions in Theorem 4.1 , it is easy to prove that $\eta_{h}$ is asymptotically exact, as shown in the following corollary.

Corollary 4.3 If, in addition to the assumptions in Theorem 4.1,

$$
\left\|\nabla\left(u-u_{h}\right)\right\|_{L^{2}(\Omega)} \geq c(u) h
$$

then

$$
\left|\frac{\eta_{h}}{\left\|\nabla\left(u-u_{h}\right)\right\|_{L^{2}(\Omega)}}-1\right| \leq C h^{\rho} .
$$

Proof. By Theorem 4.1, and the assumption in (4.7), we have

$$
\left|\frac{\eta_{h}}{\left\|\nabla\left(u-u_{h}\right)\right\|_{L^{2}(\Omega)}}-1\right| \leq \frac{\left\|\mathrm{G}^{\mathrm{h}} u_{h}-\nabla u_{h}\right\|_{L^{2}(\Omega)}}{\left\|\nabla\left(u-u_{h}\right)\right\|_{L^{2}(\Omega)}} \leq \frac{C h^{1+\rho}\|u\|_{3, \infty, \Omega}}{c(u) h}=C h^{\rho} .
$$




\section{Numerical Results}

In this section we will go over some numerical results that demonstrate the superconvergence property of $\mathrm{G}^{\mathrm{h}}$ and the asymptotic exactness of the a posteriori error estimator based on it. Also, a comparison is held between SPR and PPR with special attention paid to regions near the boundary. The results are presented through two examples in which we consider the model problem

$$
-\triangle u=f \text { in } \Omega \text {, and } u=g \text { on } \partial \Omega .
$$

Let $\mathcal{T}_{h}$ be a triangular partition of $\Omega$, and let $\mathcal{N}_{h}$ be the set of the mesh nodes in $\bar{\Omega}$. It is known that the recovery operators loose their good properties in regions near $\partial \Omega$. For that, we write $\mathcal{N}_{h}=\mathcal{N}_{h, 1} \cup \mathcal{N}_{h, 2}$, where

$$
\mathcal{N}_{h, 1}=\left\{z \in \mathcal{N}_{h}: \operatorname{dist}(z, \partial \Omega) \geq H\right\}
$$

and $H$ is some fixed positive constant. Based on that, we write $\bar{\Omega}=\Omega_{1} \cup \Omega_{2}$, where

$$
\Omega_{1}=\bigcup\left\{T \in \mathcal{T}_{h}: T \text { has all of its vertices in } \mathcal{N}_{h, 1}\right\}
$$

Let $\mathcal{A} \in \bar{\Omega}$ be the union of a set of mesh triangles in $\mathcal{T}_{h}$. The a posterior error estimator in $\mathcal{A}$ is $\eta_{h, \mathcal{A}}=\left\|R_{h} u_{h}-\nabla u_{h}\right\|_{L^{2}(\mathcal{A})}$, where $R_{h}$ denotes the recovery operator associated with SPR or PPR. To measure the accuracy of $\eta_{h, \mathcal{A}}$, we use the effectivity index $\theta_{h, \mathcal{A}}$ defined by

$$
\theta_{h, \mathcal{A}}=\frac{\eta_{h, \mathcal{A}}}{\left\|\nabla\left(u-u_{h}\right)\right\|_{L^{2}(\mathcal{A})}}
$$

One way to locally study the accuracy of the a posteriori error estimator in $\mathcal{A}$, is to use the mean, $\mu_{h, \mathcal{A}}$, and the standard deviation, $\sigma_{h, \mathcal{A}}$, of the effectivity indices defined for the mesh triangles in $\mathcal{A}$ and see how they change with $h$. If the estimator is locally exact in asymptotic sense, then $\mu_{h, \mathcal{A}} \rightarrow 1$ and $\sigma_{h, \mathcal{A}} \rightarrow 0$ as $h \rightarrow 0$. Of course

$$
\mu_{h, \mathcal{A}}=\frac{1}{N_{h, \mathcal{A}}} \sum_{T \subset \mathcal{A}} \theta_{h, T}
$$

and

$$
\sigma_{h, \mathcal{A}}^{2}=\frac{1}{N_{h, \mathcal{A}}} \sum_{T \subset \mathcal{A}}\left(\theta_{h, T}-\mu_{h, \mathcal{A}}\right)^{2},
$$

where $N_{h, \mathcal{A}}$ is the number of mesh triangles in $\mathcal{A}$.

Example 1. In this example $\Omega=(0,1)^{2}$, the solution is $u=\sin (\pi x) \sin (\pi y)$, and $H$ is taken to be $1 / 8$. For mesh generation we consider two cases. 
In the first case, the successive meshes are obtained by decomposing the unit square into $N \times N$ equal squares and then divide every square into two triangles such that the triangles are arranged in Chevron pattern. This is done for $N=16,32$, and 64 . Before we go over the results for this case, we should mention that Theorem 1.4 is not applicable and that $G^{\mathrm{h}}$ is bounded. As shown in Fig. 5, we can see that $\nabla\left(I_{h} u-u_{h}\right)$ has superconvergence that enables $\mathrm{G}^{\mathrm{h}}$ to produce superconvergent recovered gradient, as mentioned in Remark 4.2. This is not the case with SPR, as it does not preserve polynomials of order 2; a property that is crucial in proving a result similar to the one in (4.4) for the operator associated with SPR. Consequently, the behavior of the a posteriori error estimator based on SPR is inferior to that based on PPR, as shown in Fig. 6. We can see that the error estimator based on SPR is over estimating the actual error. Also, the statistics depicted in this figure shows how fast $\mu_{h, \mathcal{A}} \rightarrow 1$ and $\sigma_{h, \mathcal{A}} \rightarrow 0$ when PPR is used.

In the second case, we start with an initial mesh generated by Delauny triangulation at $h=0.1$, and in successive iterations, the new mesh is obtained from the old one by regular refinement. The results are shown in Fig. 7 and Fig. 8, where we can note two things. First, although PPR and SPR have almost the same global behavior in $\Omega_{1}$, the statistics shows that PPR is slightly better when we consider the local behavior. Secondly, the global and local properties of PPR is much better when it comes to $\Omega_{2}$.

Example 2. In this example $\Omega=(-1,1)^{2} \backslash[1 / 2,1)^{2}$. Using a polar coordinate system at $(1 / 2,1 / 2)$, the solution is taken to be $u=r^{\frac{1}{3}} \sin \left(\frac{2 \theta-\pi}{3}\right)$. As before, $H$ is $1 / 8$, and we start with an initial mesh generated with Delauny triangulation at $h=0.2$, and in successive iterations, the mesh is regularly refined. The numerical results for this example are shown in Fig. 9 and Fig. 10. As we know $\nabla u$ is singular at the reentrant corner $(1 / 2,1 / 2)$. To reduce the pollution effect due to this singularity, the region within 0.1 from $(1 / 2,1 / 2)$ is refined more than the rest of the domain in the initial mesh. Also, we expect both of PPR and SPR to behave badly near this point. Of course, this will affect the convergence rates for the recovered gradients, especially in $\Omega_{1}$, but still PPR yields some what better results. Considering the local properties, we can see that PPR is still doing better than SPR.

In conclusion, under mild conditions, we have shown that $G^{\text {h }}$ is bounded in the sense of (1.7). As a result, $\mathrm{G}^{\mathrm{h}}$ can detect any superconvergence in $\nabla\left(I_{h} u-u_{h}\right)$, and reflects it in the recovered gradient. Consequently, the a posteriori error estimator based on it is asymptotically exact, at least globally. The examples indicate that PPR is, at least, as good as SPR inside the domain, while, near the boundary, PPR seems to be superior. 


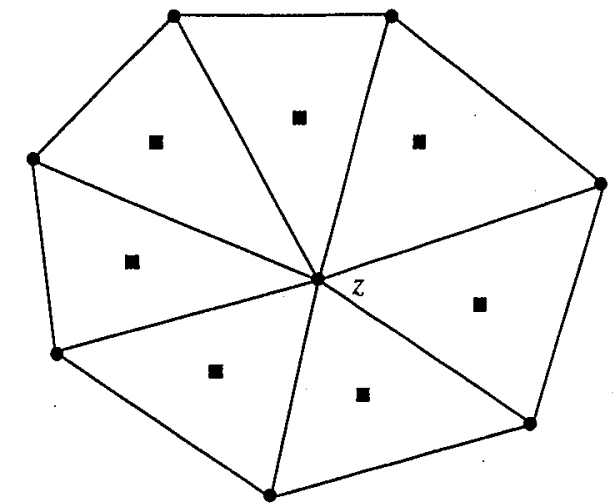

(a)

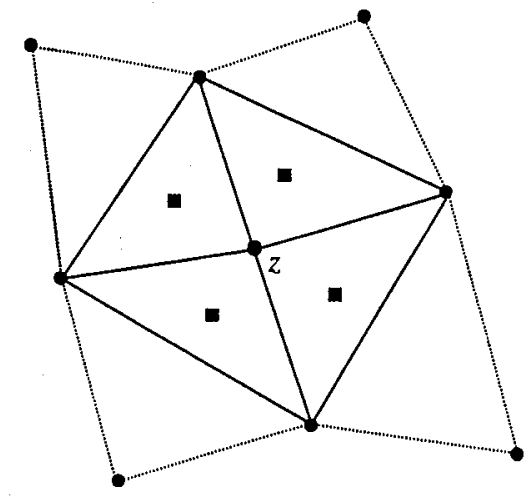

(b)

Figure 1: Patch required for gradient recovery. Sampling points for SPR are marked with while those needed for PPR are marked with •

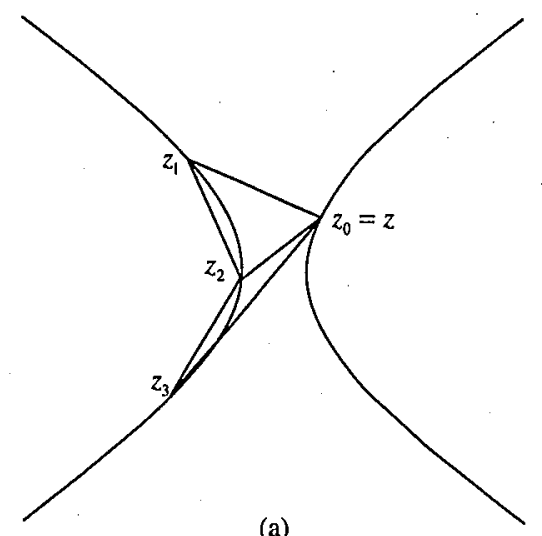

(a)

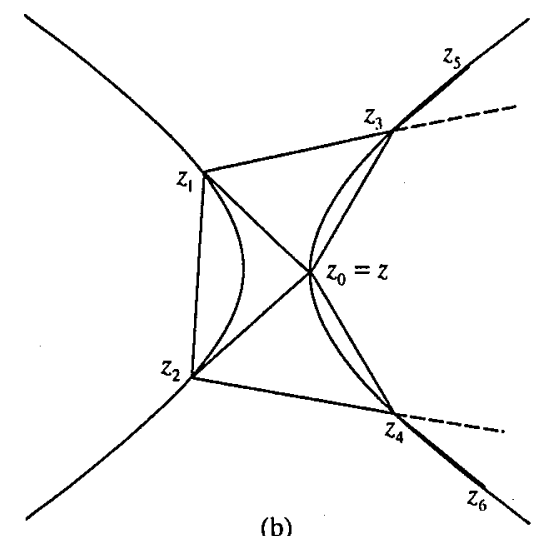

(b)

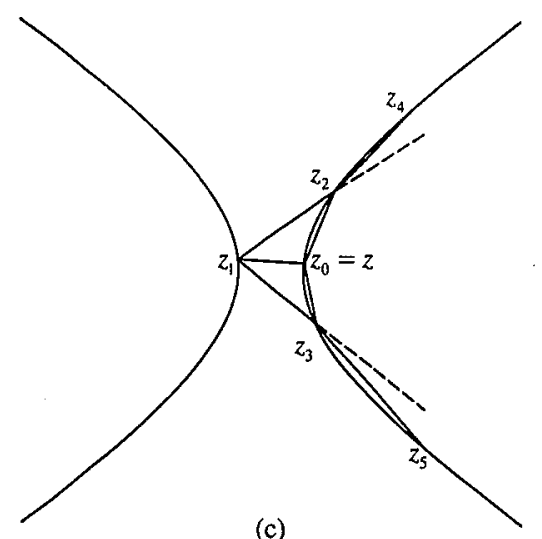

(c)

Figure 2: Nodes in $\omega_{z}$ can not be distributed on two branches of a hyperbola when $\omega_{z}$ satisfies the angle condition. 


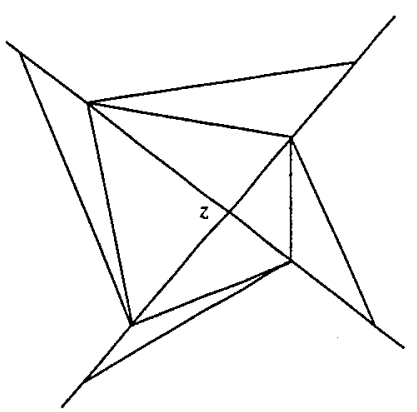

(a)

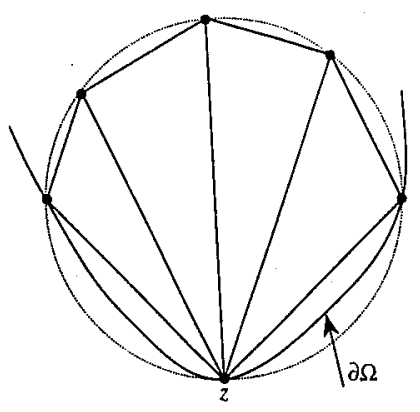

(b)

Figure 3: Patches that lead to singular $B_{z}$

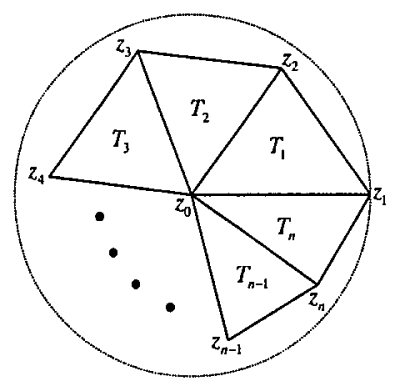

(a) $n_{1}>4$

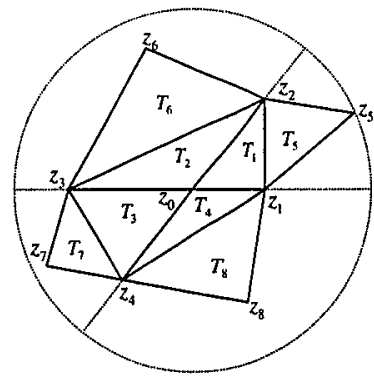

(b) $n_{1}=4$ and $n=8$

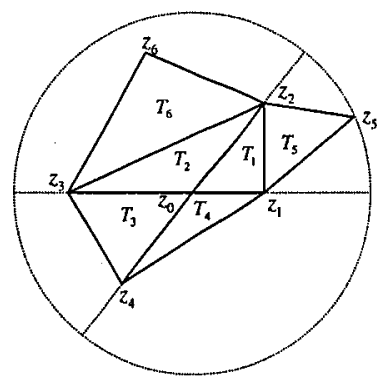

(d) $n_{1}=4$ and $n=6$

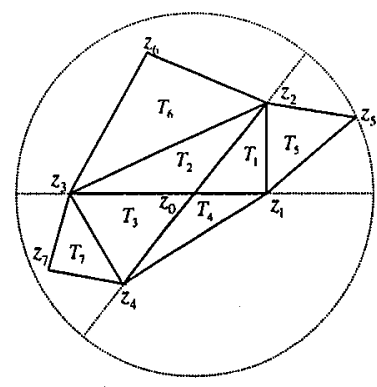

(c) $n_{1}=4$ and $n=7$

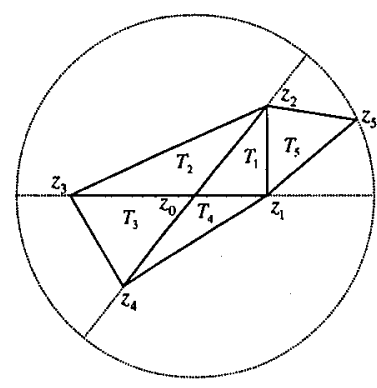

(e) $n_{1}=4$ and $n=5$

Figure 4: Triangulations for of $\overline{B(0,1)}$ for $n_{1} \geq 4$. 

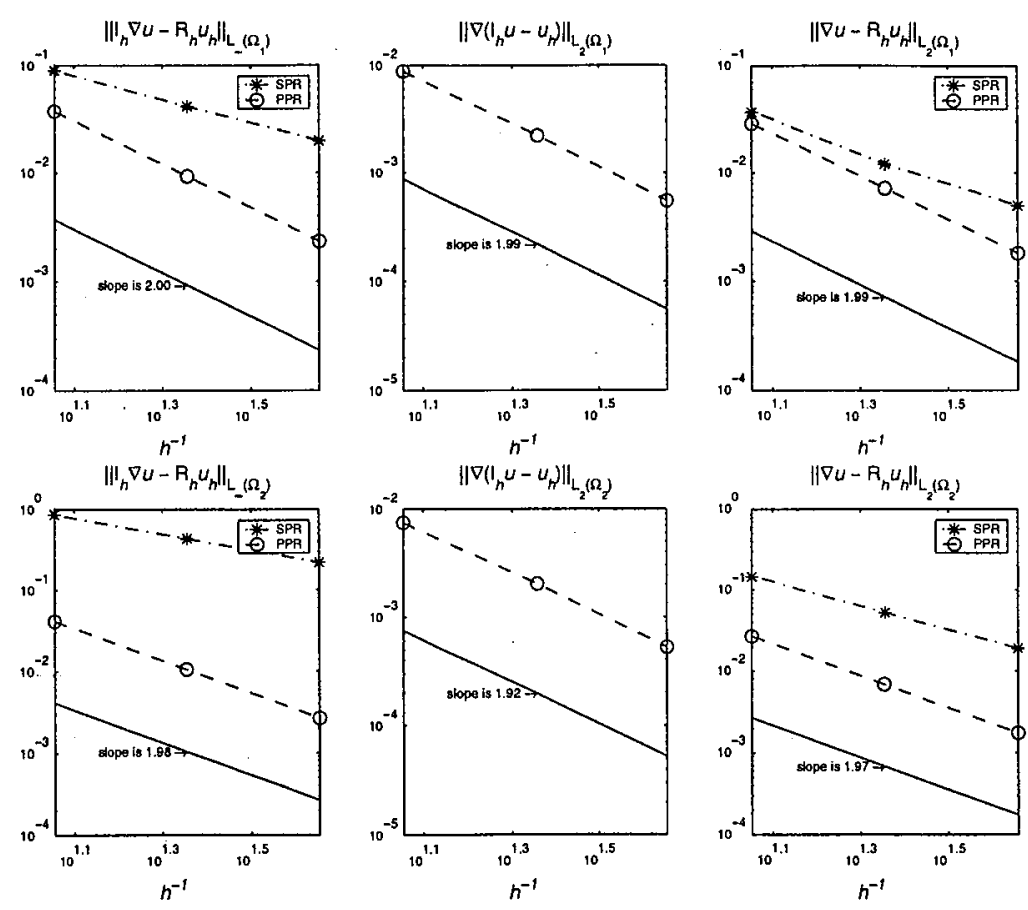

Figure 5: Convergence rates for $R_{h} u_{h}$ - Example 1 (Chevron mesh).
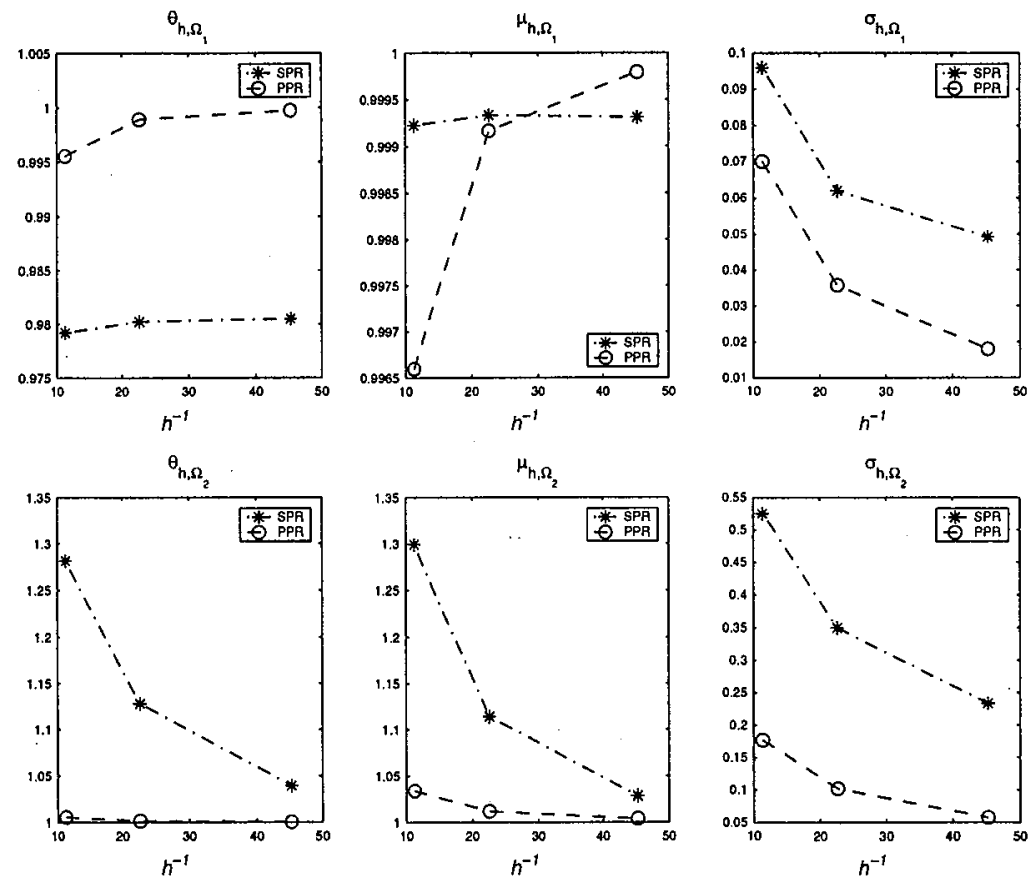

Figure 6: Properties of $\theta_{h}$ - Example 1 (Chevron mesh). 

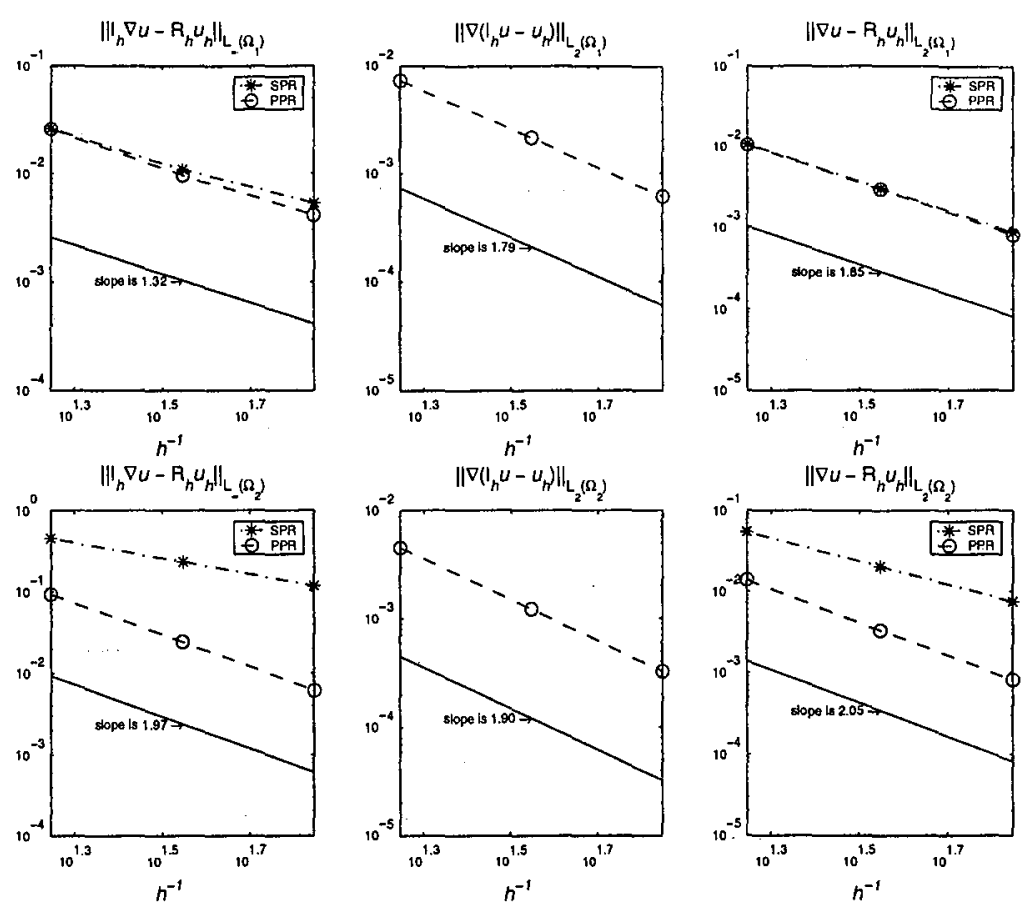

Figure 7: Convergence rates for $R_{h} u_{h}$ - Example 1 (Delauny triangulation).
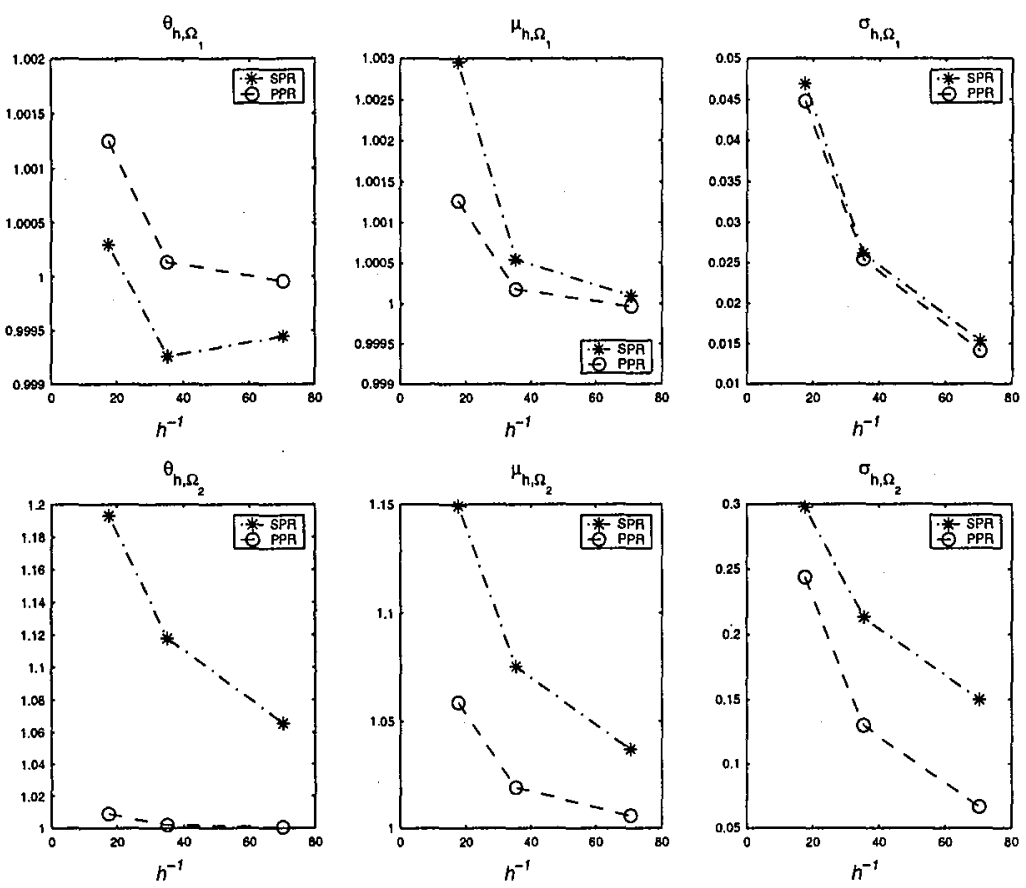

Figure 8: Properties of $\theta_{h}$ - Example 1 (Delauny triangulation). 

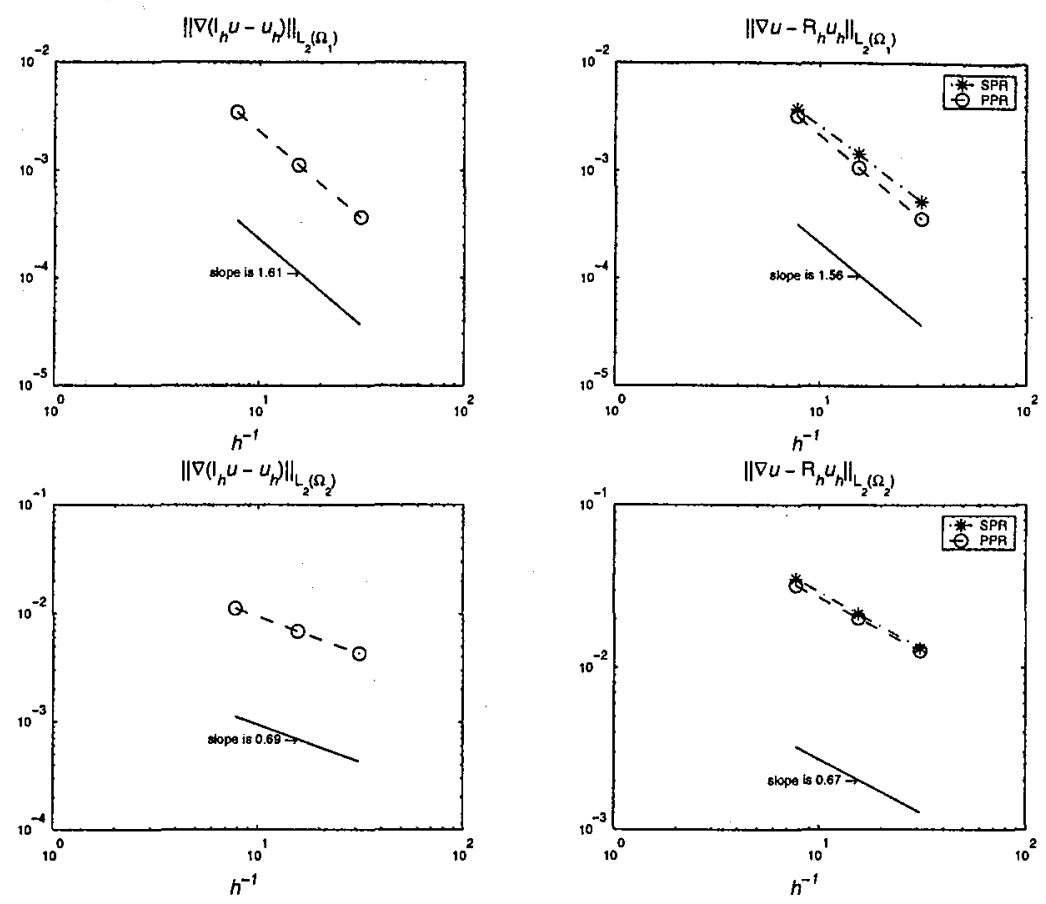

Figure 9: Convergence rates for $R_{h} u_{h}$ - Example 2.
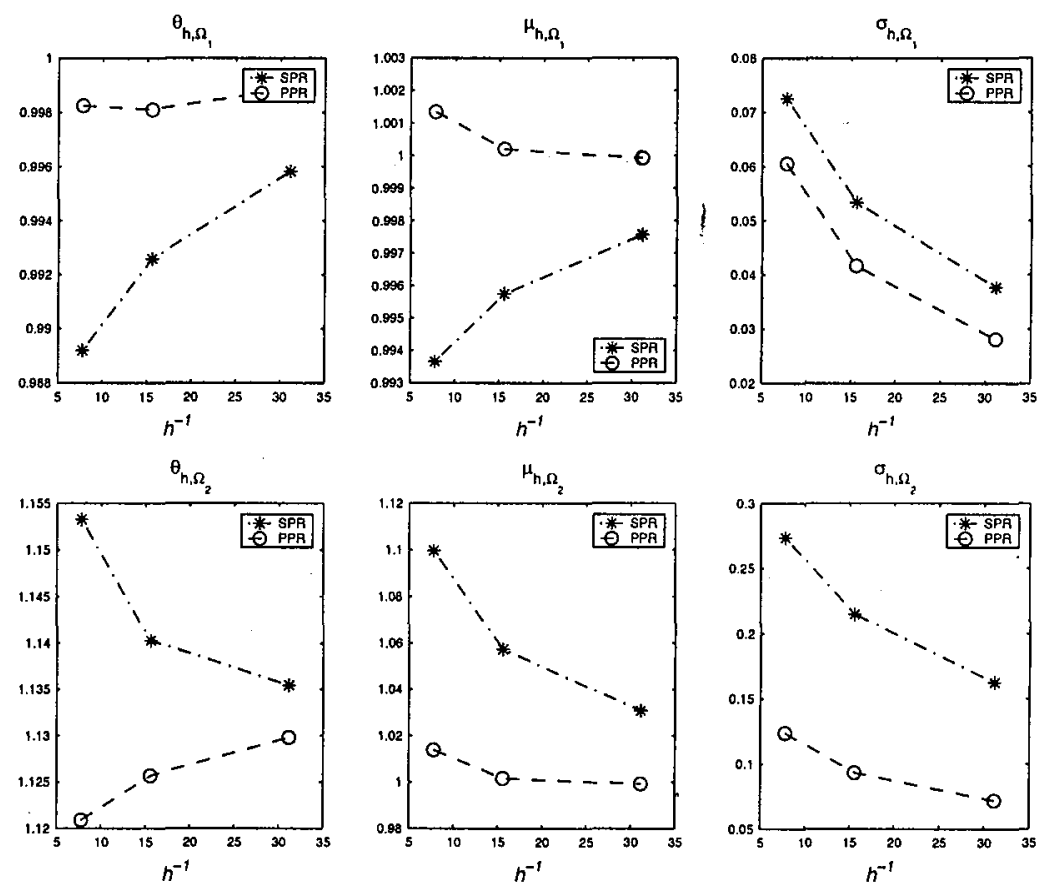

Figure 10: Properties of $\theta_{h}$ - Example 2. 


\section{References}

[1] Ainsworth, M., Oden, J.T., A POSERIORI ERROR ESTIMATION IN FINITE ELEMENT ANALYSIS, Wiley Interscience, New York, 2000, pp. 73-77.

[2] Babuška, I., Aziz, A.K., On the angle condition in the finite element method, SIAM J. Numer. Anal., 13 (1976), pp. 214-226.

[3] Babuška, I., Strouboulis, T., THE FINITE ELEMENT METHOD AND ITS RELIABILITY, Oxford University Press, London, 2001.

[4] Bank, R.E., Weiser, A., Some a posteriori error estimators for elliptic partial differential equations, Math. Comp., 44 (1985), pp.283-301.

[5] Bank, R.E., Xu, J., Asymptotically exact a posteriori error estimators, part I: grids with superconvergence, (submitted for review)

[6] Blum, H., Lin, Q., Rannacher, R., Asymptotic error expansion and richardson extrapolation for linear finite elements, Numer. Math., 49 (1986), no. 1, pp. 11-37.

[7] Li, X.D, Wiberg, N.-E., A posteriori error estimate by element patch postprocessing, adaptive analysis in energy norm and $L_{2}$ norms, Comput. \& Structures, 53 (1994), no. 4, pp. 907-919.

[8] McLeod, Robin J.Y., Baart, M. Louisa, GEMOETRY AND INTERPOLATION OF CURVES AND SURFACES, Cambridge University Press, 1998, pp. 41.

[9] Wiberg, N.-E., Li, X.D, Superconvergent patch recovery of finite element solution and a posteriori error $L_{2}$ norm estimate, Comm. Numer. Methods Eng., 10 (1994), no. 4, pp. 313-320.

[10] Xu, J., Zhang, Z. , Analysis of recovery type a posteriori error estimators for mildly structured grids, Research Report \#7 (2002), Department of Mathematics, Wayne State University.

[11] Zhang, Z., Naga, A., A meshless gradient recovery method, part I: superconvergence property, Research Report \#2 (2002), Department of Mathematics, Wayne State University.

[12] Zienkiewicz, O.C., Zhu, J.Z., A simple error estimator and adaptive procedure for practical engineering analysis, Internat. J. Numer. Methods Eng, 24 (1987), pp. 337-357. 
[13] Zienkiewicz, O.C., Zhu, J.Z., The superconvergence patch recovery and a posteriori error estimates, part I: the recovery technique, Internat. J. Numer. Methods Eng, 33 (1992), pp. 1331-1364.

[14] Zienkiewicz, O.C., Zhu, J.Z., The superconvergence patch recovery and a posteriori error estimates, part II: error estimates and adaptivity, Internat. J. Numer. Methods Eng, 33 (1992), 1365-1382. 